\title{
Embedding Effect on the Mechanical Stability of Pressurised Carbon Nanotubes
}

\author{
Motohiro Sato, ${ }^{1}$ Hisao Taira, ${ }^{1,2}$ Tetsuro Ikeda, ${ }^{1}$ and Hiroyuki Shima ${ }^{3}$ \\ ${ }^{1}$ Division of Engineering and Policy for Sustainable Environment, Faculty of Engineering, Hokkaido University, \\ Sapporo 060-8628, Japan \\ ${ }^{2}$ Department of Physics, Faculty of Science, Hokkaido University, Sapporo 060-0810, Japan \\ ${ }^{3}$ Department of Environmental Sciences \& Interdisciplinary Graduate School of Medicine and Engineering, \\ University of Yamanashi, 4-4-37 Takeda, Kofu, Yamanashi 400-8510, Japan
}

Correspondence should be addressed to Hiroyuki Shima; hshima@yamanashi.ac.jp

Received 6 March 2013; Revised 15 April 2013; Accepted 26 April 2013

Academic Editor: Teng Li

Copyright (C) 2013 Motohiro Sato et al. This is an open access article distributed under the Creative Commons Attribution License, which permits unrestricted use, distribution, and reproduction in any medium, provided the original work is properly cited.

\begin{abstract}
We elaborate on the cross-sectional deformation of carbon nanotubes embedded into a self-contracting host medium. The continuum elastic approach is used to formulate the mechanical energy of both the embedded nanotubes and the self-contracting outer medium with finite thickness. Our formula allows us to evaluate the critical radial pressure applied on the interface between the embedded nanotube and the outer contracting medium as well as the deformation mode that arises immediately above the critical pressure. An interesting mechanical implication of the embedding effect, that is, the power-law dependence of the critical pressure on the elastic modulus of the medium, is deduced by the theoretical approach established.
\end{abstract}

\section{Introduction}

The salient structural feature of a carbon nanotube is its selfrepairing behaviour that arises under high-energy beam irradiation $[1,2]$. When the kinetic energy transferred from the incident beam to the constituent carbon atoms is sufficiently large, the atoms are pushed away from the original equilibrium positions, leaving vacancies in the host hexagonal lattice $[3,4]$. In typical solids, such irradiation-induced vacancies survive without curing as time passes. However, this is not the case in carbon nanotubes; the removal of carbon atoms from the purely hexagonal lattice leads to a local reconstruction that acts to maintain its coherent network structure with cylindrical geometry. For this reason, carbon nanotubes are often referred to as self-repairing (or self-healing) materials [5-7].

This self-repairing nature provides beneficial effects for manipulating the carbon nanotube morphology, especially when combined with heat treatment. For instance, finetuning of electron beam irradiation makes it possible to synthesize multiwall carbon nanotubes (MWNTs) with reduced interwall spacings [8]; the spontaneous shrinkage in the radial direction is a result of the knock-on collision of carbon atoms followed by annealing reconstruction of the vacancies. This experimental finding implies that when the outermost carbon walls of an MWNT are eroded selectively by irradiation, the self-contraction of the outermost walls exerts high pressure on the encapsulated, undamaged innermost walls [9-11]. Application of high pressure may then trigger a novel class of cross-sectional transformations of the inner walls [12], similar to the case of pristine (irradiation-free) MWNTs under hydrostatic pressure $[13,14]$. Another class of radial contraction has been observed in MWNTs synthesized in the presence of nitrogen [15]. The yielded nanotubes showed polygon-shaped cross-sections rather than ordinary circular ones, a phenomenon that is partly attributed to the interwall thermal contraction upon cooling, as verified numerically by molecular dynamics simulations [16].

From a nanoengineering perspective, the tunability of cross-sectional geometry may be useful for developing nanofluidic [17-19] or nanoelectrochemical devices [20] based on carbon nanotubes, because both utilize the hollow cavity within the innermost tube. A very interesting issue from an academic viewpoint is the effect of the core tube 
deformation on the physicochemical properties of intercalated molecules confined in the hollow cavity. It is indeed known that various types of intercalated molecules can fill the innermost hollow cavities of nanotubes [18] and exhibit intriguing behaviours that are distinct from their macroscopic counterparts [21-23]. These distinct behaviours originate from the similarity between the intermolecular spacings and the linear dimension of the nanoscale compartment. Therefore, cross-sectional deformation that breaks cylindrical symmetry will provide a clue to improving the performance of nanotube-based devices.

In this paper, we establish the continuum elastic approach that describes the cross-sectional deformation of carbon nanotubes surrounded by a self-contracting host medium. The mechanical energy of both the pressurized nanotubes and the contracting medium with finite thickness are formulated using thin-shell theory. The obtained formula allows us to evaluate the critical radial pressure applied on the interface between the inner nanotube and the outer medium. Our numerical calculations have unveiled a power-law dependence of the critical pressure on the elastic modulus of the medium that is independent of the medium thickness.

\section{Methodology}

2.1. Relevant Energy Components. Figure 1 illustrates the selfcontraction process of MWNTs subjected to high-energetic beam irradiation. Under irradiation, the induced beam kicks off a portion of carbon atoms at outer walls, causing vacancies followed by spontaneous shrinkage as marked by color in Figure 1(b). Even during the outer-walls self-contraction, the inner walls remain undamaged by irradiation, and thus they tend to keep their initial tube radii. As a result, the contracting outerwalls exert a high pressure, designated by $p$, on the inner undamaged walls (see Figure 1). A possible consequence of the radiation-induced high-pressure application is a circumferentially wrinkling structure, called radial corrugation [13], in the inner undamaged walls embedded in the eroded region, as examined later in an approximation based on the thin-shell theory.

The stable cross-sectional shape of the embedded tube is obtained by minimizing its mechanical energy $U$ per unit axial length [13] as

$$
U=U_{D}+U_{I}+U_{m}+\Omega
$$

The first term $U_{D}=\sum_{i=1}^{N} U_{D}^{(i)}$ with the definition

$$
U_{D}^{(i)}=\frac{r_{i}}{2}\left(\frac{C}{1-v^{2}} \int_{0}^{2 \pi} \mathscr{I}_{i}^{2} d \theta+D \int_{0}^{2 \pi} \mathscr{B}_{i}^{2} d \theta\right)
$$

represents the deformation energy of the embedded nanotubes. $\mathscr{I}_{i}$ and $\mathscr{B}_{i}$ are, respectively, in-plane and bendinginduced strains of the $i$ th wall, and $\theta$ is the circumferential coordinate. For (2), we supposed that each $i$ th wall had a radius $r_{i}$ prior to cross-sectional deformation and that the deformation caused a displacement $\mathbf{x}_{i}=\left(u_{i}, v_{i}\right)$ of a volume element of the $i$ th wall at $\left(r_{i}, \theta\right)$ in the polar coordinate

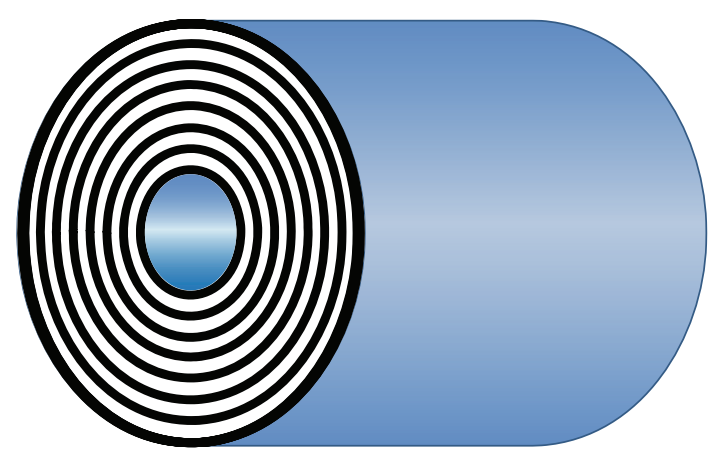

(a) MWNT before irradiation

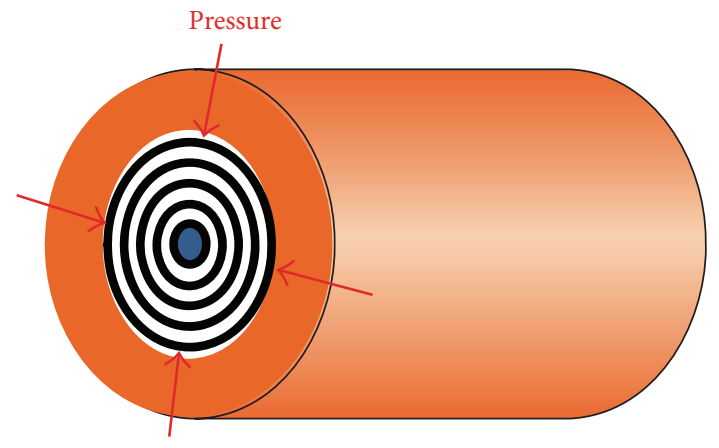

(b) MWNT after irradiation

FIgURE 1: Schematics of the pressurized carbon nanotubes. (a) A pristine MWNT prior to high-energy beam irradiation. (b) An irradiated MWNT whose outermost carbon walls contract radially because of the self-repairing property of the eroded region (indicated in orange).

representation. The two strain terms $\mathscr{I}_{i}$ and $\mathscr{B}_{i}$ are described in terms of the displacement components by [14]

$$
\mathscr{I}_{i}=\frac{u_{i}+\partial_{\theta} v_{i}}{r_{i}}+\frac{1}{2}\left(\frac{\partial_{\theta} u_{i}-v_{i}}{r_{i}}\right)^{2}, \quad \mathscr{B}_{i}=-\frac{\partial_{\theta}^{2} u_{i}-\partial_{\theta} v_{i}}{r_{i}^{2}},
$$

where $\partial_{\theta}=\partial / \partial \theta$.

For quantitative discussions, the elastic coefficients $C$ and $D$ need to be carefully determined. In conventional thin-shell theory for macroscopic objects, $C$ and $D$ are related to the Young's modulus $E$ of the wall and its thickness $h$ as

$$
C=E h, \quad D=\frac{E h^{3}}{12\left(1-v^{2}\right)} \quad \text { for macroscopic shells. }
$$

However, for carbon nanotubes, the macroscopic relations for $C$ and $D$ noted earlier fail because there is no unique way of defining the thickness of the graphene wall [24]. Thus, the values of $C$ and $D$ should be evaluated $a b$ initio from direct measurements or computations of carbon sheets, without reference to the macroscopic relations. In actual calculations, we substitute $C=345 \mathrm{nN} / \mathrm{nm}, D=0.238 \mathrm{nN} \cdot \mathrm{nm}$ for carbon nanotubes, and $v=0.149$ from prior work [25] based on the density functional theory.

The second term, $U_{I}=\sum_{i, j=i \pm 1} U_{I}^{(i, j)}$, in (1) accounts for the van der Waals (vdW) interaction energy, which 
determines the equilibrium distance between adjacent concentric walls. Thus far, several continuum models for the $\mathrm{vdW}$ interactions have been proposed. Expressions for the wall-wall interaction proposed in [26] were based on the surface integration of the vdW force and its derivative over the cylindrical walls, while disregarding the vectorial nature of the force. The significance of the vectorial nature of the force was addressed in [27], where analytical expressions for the wall-wall interaction were obtained by considering only the component of the vdW force normal to the wall. In accordance with the result of [27], we define the interaction energy by

$$
U_{I}^{(i, j)}=\frac{c_{i j}\left(r_{i}+r_{j}\right)}{4} \int_{0}^{2 \pi}\left(u_{i}-u_{j}\right)^{2} d \theta,
$$

where the coefficients $c_{i j}$ are derived through a harmonic approximation of the interwall force [28] associated with the $\mathrm{vdW}$ intermolecular potential

$$
V(r)=4 \alpha\left[\left(\frac{\beta}{r}\right)^{12}-\left(\frac{\beta}{r}\right)^{6}\right]
$$

with the definitions of $\alpha=2.39 \mathrm{meV}$ and $\beta=0.3415 \mathrm{~nm}$ the same as those in [29]. Equation (5) takes into account correctly the normal-to-wall component of vdW forces, and it is valid for infinitesimal deformation, which we address in the present work.

The final term $\Omega$ in (1) is the negative of the work done by $p$ during cross-sectional deformation; it can be written as [13]

$$
\Omega=p \int_{0}^{2 \pi}\left(r_{N} u_{N}+\frac{u_{N}^{2}+v_{N}^{2}-v_{N} \partial_{\theta} u_{N}+u_{N} \partial_{\theta} v_{N}}{2}\right) d \theta .
$$

Note that all three terms are functions of $u_{i}(p, \theta)$ and $v_{i}(p, \theta)$ of the $i$ th wall under $p$.

The remaining term, $U_{m}$, in (1) is the elastic energy of the eroded medium surrounding the inner part of the nanotubes. To derive it, we assume that the medium has finite thickness of $r_{m}-r_{N}$, where $r_{m}$ is the outmost radius of the cylindershaped surrounding medium and $r_{N}$ is the outmost tube radius of the embedded nanotube. In addition, the medium is assumed to be homogeneous and isotropic with Young's modulus $E_{m}$ and Poisson's ratio $v_{m}$. The validity of the latter assumption depends on the following two effects of irradiation on the mechanical stiffness of MWNTs. Irradiation reduces the axial stiffness because it creates vacancies [30, 31], and it simultaneously enhances the radial stiffness, owing to the production of covalent bonding between adjacent walls [32]. The possible value of $E_{m}$ ranges from $100 \mathrm{GPa}$ (for amorphous carbon) $[33,34]$ to much less. An explicit form of $U_{m}$ is presented in the next section.

2.2. Corrugation Mode Analysis. Our objectives are to determine (i) the optimal displacements $u_{i}$ and $v_{i}$ that minimize $U$ under a given value of $p$ and (ii) the critical pressure $p_{c}$ above which the circular cross section of the embedded nanotube is elastically deformed into a noncircular one. These are accomplished by decomposing the displacement $u_{i}(p, \theta)$ just after buckling as follows:

$$
u_{i}(p, \theta)=u_{i}^{(0)}(p)+\delta u_{i}(\theta)
$$

In (8), the term $u_{i}^{(0)}(p) \propto p$ describes a uniform radial contraction at $p<p_{c}$, at which the cross section remains circular and thus the displacement is independent of $\theta$. The other term $\delta u_{i}(\theta)$ describes a deformed (noncircular) cross-section observed immediately above $p_{c}$. Note that the superscript (0) attached to $u_{i}^{(0)}(p)$ differentiates it from the $\theta$-dependent displacement $u_{i}(p, \theta)$ observed at $p>p_{c}$. As to $v_{i}(p, \theta)$, we can write

$$
v_{i}(p, \theta)=\delta v_{i}(\theta),
$$

because no circumferential displacement arises at $p<p_{c}$ (i.e., $\left.v_{i}^{(0)}(p) \equiv 0\right)$.

Applying the variation method to $U$ with respect to $u_{i}$ and $v_{i}$, we obtain a system of $2 N$ linear differential equations with regard to $\delta u_{i}(\theta)$ and $\delta v_{i}(\theta)$. To derive the $2 N$ linear differential equations, quadratic or cubic terms in $\delta u_{i}$ and $\delta v_{i}$ are omitted since we consider elastic deformation with sufficiently small displacements. In addition, the terms consisting only of $u_{i}^{(0)}$ and $p$ are also omitted; the sum of such terms should be equal to zero since $u_{i}^{(0)}$ represents an equilibrium circular cross-section under $p$. In fact, the function form of $u_{i}^{(0)}(p)$ is determined by the fact that the sum of those terms equals zero. The differential equations can be solved using the Fourier series expansions

$$
\delta u_{i}(\theta)=\sum_{n=1}^{\infty} \delta \bar{\mu}_{i}^{(n)} \cos n \theta, \quad \delta v_{i}(\theta)=\sum_{n=1}^{\infty} \delta \bar{v}_{i}^{(n)} \sin n \theta,
$$

wherein we took into account that $\delta u_{i}, \delta v_{i}$, and their derivatives are periodic in $\theta$. Substituting the expansions into the differential equations results in the matrix equation $\mathbf{M u}=\mathbf{0}$; the vector $\mathbf{u}$ consists of $\delta \bar{\mu}_{i}^{(n)}$ and $\delta \bar{\nu}_{i}^{(n)}$ with all possible $i$ and $n$, and the matrix $\mathbf{M}$ involves one variable $p$ and other material parameters. It should be noted that, due to the orthogonality of $\cos n \theta$ and $\sin n \theta$, the matrix $\mathbf{M}$ can be expressed by a block diagonal matrix of the form

$$
\mathbf{M}=\mathbf{M}_{n=1} \oplus \mathbf{M}_{n=2} \oplus \cdots .
$$

Here, $\mathbf{M}_{n=k}$ for arbitrary integer $k$ is a $2 N \times 2 N$ submatrix that satisfies $\mathbf{M}_{n=k} \mathbf{u}_{n=k}=\mathbf{0}$, where $\mathbf{u}_{n=k}$ is a $2 N$-column vector composed of $\delta \bar{\mu}_{i}^{(k)}$ and $\delta \bar{v}_{i}^{(k)}$. As a result, the secular equation $\operatorname{det}(\mathbf{M})=0$ is rewritten by

$$
\operatorname{det}\left(\mathbf{M}_{n=1}\right) \operatorname{det}\left(\mathbf{M}_{n=2}\right) \cdots=0 .
$$

Solving (12) with respect to $p$, we obtain a sequence of discrete values of $p$. Among these values, the minimum one serves as the critical pressure $p_{c}$ that is associated with a specific integer $k$. From the definition, the $p_{c}$ associated with a specific $k$ allows only $\delta \bar{\mu}_{i}(n=k)$ and $\delta \bar{\nu}_{i}(n=k)$ be finite, but it requires $\delta \bar{\mu}_{i}(n \neq k) \equiv 0$ and $\delta \bar{v}_{i}(n \neq k) \equiv 0$. Immediately 
above $p_{c}$, therefore, the cross section of embedded nanotubes becomes radially deformed as described by

$$
u_{i}(\theta)=u_{i}^{(0)}\left(p_{c}\right)+\delta \bar{\mu}_{i}^{(k)} \cos k \theta, \quad v_{i}(\theta)=\delta \bar{v}_{i}^{(k)} \sin k \theta
$$

where the value of $k$ is uniquely determined by the one-toone relation between $k$ and $p_{c}$.

\section{Mechanical Energy of the Embedding Medium}

3.1. Total Energy Cost. In polar coordinates, the radial and circumferential components of normal stress in the medium are denoted by $\sigma_{r}$ and $\sigma_{\theta}$, respectively, and the shear stress is denoted by $\tau_{r \theta}$; all three quantities are functions of $r$ and $\theta$. Then, $U_{m}$ is determined by $\sigma_{r}$ and $\tau_{r \theta}$ at $r=r_{N}$ as

$$
\begin{gathered}
U_{m}=U_{m}^{(0)}+\Delta U_{m}^{(n)} \\
U_{m}^{(0)}=\left.\frac{r_{N}}{2} \int_{0}^{2 \pi} \sigma_{r}^{(0)}\right|_{r=r_{N}} u_{N}^{(0)} d \theta \\
\Delta U_{m}^{(n)}=\frac{r_{N}}{2} \int_{0}^{2 \pi}\left(\left.\sigma_{r}^{(n)}\right|_{r=r_{N}} \delta u_{N}+\left.\tau_{r \theta}^{(n)}\right|_{r=r_{N}} \delta v_{N}\right) d \theta
\end{gathered}
$$

where $\delta u_{N}$ and $\delta v_{N}$ describe the corrugation amplitudes of the outermost wall of the embedded nanotube; see (10). The superscripts $(0)$ and $(n)$ indicate the quantities corresponding to uniform contraction and radial corrugation, respectively. In other words, $U_{m}^{(0)}$ represents the energy required for uniform radial contraction of the surrounding medium keeping in contact with the embedded nanotube, and $\Delta U_{m}$ represents the energy required for radial corrugation with mode index $n$.

3.2. Stress-Strain-Displacement Relation. The mechanics of an elastic medium is governed by the stress function $\phi$, which satisfies the so-called compatibility equation [35]

$$
\left(\partial_{r}^{2}+r^{-1} \partial_{r}+r^{-2} \partial_{\theta}\right)^{2} \phi(r, \theta)=0,
$$

where $\partial_{r}=\partial / \partial r$ and $\partial_{\theta}=\partial / \partial \theta$. Once $\phi$ is obtained, we can deduce the stress components as follows:

$$
\begin{gathered}
\sigma_{r}=\left(r^{-1} \partial_{r}+r^{-2} \partial_{\theta}^{2}\right) \phi, \quad \sigma_{\theta}=\partial_{r}^{2} \phi \\
\tau_{r \theta}=\partial_{r}\left(r^{-1} \partial_{\theta}\right) \phi .
\end{gathered}
$$

By definition, the strain components $\varepsilon_{r}, \varepsilon_{\theta}$, and $\gamma_{r \theta}$ are given by the matrix form

$$
\begin{gathered}
{\left[\begin{array}{c}
\varepsilon_{r} \\
\varepsilon_{\theta} \\
\gamma_{r \theta}
\end{array}\right]=\mathbf{G}\left[\begin{array}{c}
\sigma_{r} \\
\sigma_{\theta} \\
\tau_{r \theta}
\end{array}\right]=\left[\begin{array}{cc}
\partial_{r} & 0 \\
r^{-1} & r^{-1} \partial_{\theta} \\
r^{-1} \partial_{\theta} & \partial_{r}-r^{-1}
\end{array}\right]\left[\begin{array}{l}
u \\
v
\end{array}\right],} \\
\mathbf{G}=\frac{1}{E_{m}}\left[\begin{array}{ccc}
1-v_{m}^{2} & -v_{m}\left(1+v_{m}\right) & 0 \\
-v_{m}\left(1+v_{m}\right) & 1-v_{m}^{2} & 0 \\
0 & 0 & 2\left(1+v_{m}\right)
\end{array}\right],
\end{gathered}
$$

where $u=u(r, \theta)$ and $v=v(r, \theta)$ are, respectively, the radial and circumferential displacements of a volume element in the elastic medium.

The general solution of (17) is given by

$$
\phi(r, \theta)=\sum_{n=0}^{\infty} \phi_{n}(r, \theta)=\sum_{n=0}^{\infty}\left[f_{n}(r) \cos n \theta+g_{n}(r) \sin n \theta\right]
$$

The zeroth component $\phi_{0}$ represents a uniform contraction of the circular cross section, thus corresponding to the energy $U_{m}^{(0)}$ that we have introduced in (15). The first one, $\phi_{1}$, implies a rigid-body translation, which is irrelevant to our consideration. Other components, $\phi_{n}$ for $n \geq 2$, describe radial corrugations with mode index $n$, thus providing the energy $\Delta U_{m}^{(n)}$ given by (16). In the following, we set [36]

$$
\begin{gathered}
f_{0}=a_{0} \log r+c_{0} r^{2} \\
f_{n}=a_{n} r^{-n}+b_{n} r^{2-n}+c_{n} r^{2+n}+d_{n} r^{n}, \quad(n \geq 2)
\end{gathered}
$$

in order to obtain physically relevant solutions of $\sigma_{r}, \sigma_{\theta}$, and $\tau_{r \theta}$ that decay with increasing $r$. Without loss of generality, we set $g_{n} \equiv 0$ in (21) according to our assumption of cosine-type radial displacement $\delta u_{i}(\theta)$; see (10). We emphasize that the hypothesized solution forms of $f_{0}$ and $f_{n \geq 2}$ differ from those in our earlier work [12]; in the present study, we introduce the positive power terms of $r$ in (22) to take into account correctly the boundary condition at $r=r_{m}$, whereas these terms were omitted in [12].

3.3. Energy Cost under Uniform Contraction. We now evaluate the explicit form of the energy $U_{m}^{(0)}$. It results from the uniform contraction of the medium described by the $r$ dependent medium displacement $u^{(0)}(r)$. The elastic nature of the embedding medium assures that

$$
\sigma_{r}^{(0)}(r)=\kappa(r) u^{(0)}(r)
$$

with the $r$-dependent stiffness coefficient $\kappa(r)$. In addition, complete contact at the interface of the medium and the embedded nanotube implies

$$
u^{(0)}\left(r_{N}\right)=u_{N}^{(0)}
$$

Hence, the previous expression of $U_{m}^{(0)}$, given in (15), can be rewritten as

$$
U_{m}^{(0)}=\frac{\kappa_{0}}{2} \int_{0}^{2 \pi}\left\{u_{N}^{(0)}\right\}^{2} r_{N} d \theta \quad \text { with } \kappa_{0} \equiv \kappa\left(r_{N}\right) .
$$

The remaining task is, therefore, to represent $\kappa_{0}$ in terms of already-known material parameters such as $E_{m}, v_{m}, r_{m}$, and $r_{N}$

To accomplish this task, we consider a specific solution of (17) that has the form $\phi=\phi_{0}$, and then we substitute it back into (18) to obtain

$$
\sigma_{r}^{(0)}=a_{0} r^{-2}+2 c_{0}, \quad \sigma_{\theta}^{(0)}=-a_{0} r^{-2}+2 c_{0},
$$


and $\tau_{r \theta}^{(0)}=0$. The coefficients $a_{0}$ and $c_{0}$ are determined by imposing the boundary conditions of $\sigma_{r}^{(0)}$ at $r=r_{N}$ and $r=$ $r_{m}$ as follows:

$$
a_{0} r_{N}^{-2}+2 c_{0}=p, \quad a_{0} r_{m}^{-2}+2 c_{0}=0
$$

Eliminating $a_{0}$ and $c_{0}$ from (27), we have

$$
\begin{aligned}
& \sigma_{r}^{(0)}(r)=\frac{r_{N}^{2} p}{r_{m}^{2}-r_{N}^{2}}\left(\frac{r_{m}^{2}}{r^{2}}-1\right), \\
& \sigma_{\theta}^{(0)}(r)=\frac{-r_{N}^{2} p}{r_{m}^{2}-r_{N}^{2}}\left(\frac{r_{m}^{2}}{r^{2}}+1\right) .
\end{aligned}
$$

The obtained $\sigma_{r}^{(0)}(r)$ and $\sigma_{\theta}^{(0)}(r)$ lead to the displacements

$$
u^{(0)}(r)=-\frac{1+v_{m}}{E_{m}} \cdot \frac{r_{N}^{2} p}{r_{m}^{2}-r_{N}^{2}}\left[\left(1-2 v_{m}\right) r+\frac{r_{m}^{2}}{r}\right]
$$

and $v^{(0)}(r)=0$ at the medium region $\left(r_{N}<r<r_{m}\right)$, as confirmed by (19). By comparing (23), (28), and (29), we conclude that

$$
\kappa_{0}=-\frac{E_{m}}{\left(1+v_{m}\right)} \cdot\left(\frac{r_{m}^{2}}{r_{N}^{2}}-1\right) \cdot\left[\left(1-2 v_{m}\right) r_{N}+\frac{r_{m}^{2}}{r_{N}}\right]^{-1} .
$$

3.4. Energy Cost under Radial Corrugation. Next, we consider the energy $\Delta U_{m}^{(n)}(n \geq 2)$ that corresponds to the radial corrugation of the $n$th order. A similar procedure to the case of $n=0$ yields

$$
\begin{aligned}
\sigma_{r}^{(n)} & (r, \theta) \\
= & \left\{-n(n+1) a_{n} r^{-2}-(n-1)(n+2) b_{n}\right\} r^{-n} \cos n \theta \\
& +\left\{-n(n-1) d_{n} r^{-2}-(n+1)(n-2) c_{n}\right\} r^{n} \cos n \theta, \\
\sigma_{\theta}^{(n)} & (r, \theta) \\
= & \left\{n(n+1) a_{n} r^{-2}+(n-1)(n-2) b_{n}\right\} r^{-n} \cos n \theta \\
& +\left\{n(n-1) d_{n} r^{-2}+(n+1)(n+2) c_{n}\right\} r^{n} \cos n \theta, \\
\tau_{r \theta}^{(n)} & (r, \theta) \\
= & \left\{n(n+1) a_{n} r^{-2}+n(n-1) b_{n}\right\} r^{-n} \sin n \theta \\
& -\left\{n(n-1) d_{n} r^{-2}+n(n+1) c_{n}\right\} r^{n} \sin n \theta,
\end{aligned}
$$

\section{Results and Discussions}

Figure 2 shows the critical pressure $p_{c}$ required for the cross-sectional deformation of embedded carbon nanotubes. Different types of curves in the plots (dashed, dashed-dotted, and solid) correspond to different values of Young's modulus ratio $E_{m} / E$, where $E=1 \mathrm{TPa}$ is assumed to be the nanotube's modulus. We found that, independent of the $E_{m} / E$ value, the $p_{c}$ curves are upward convex as functions of the medium 


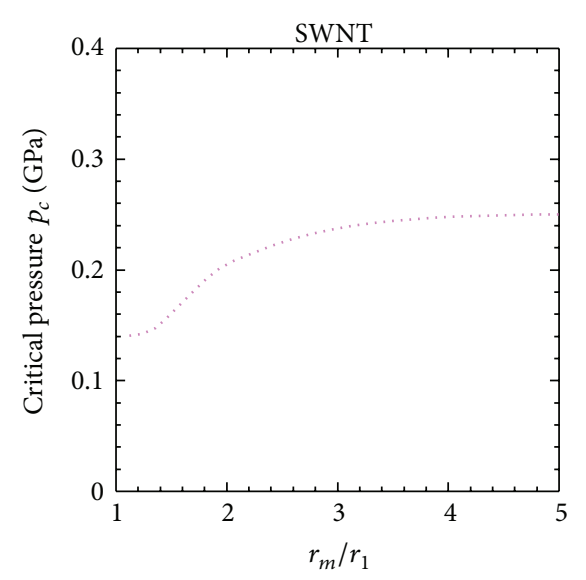

(a)

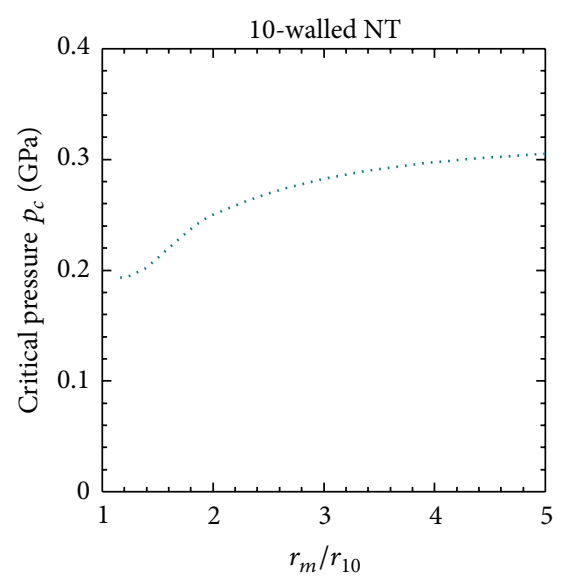

(d)

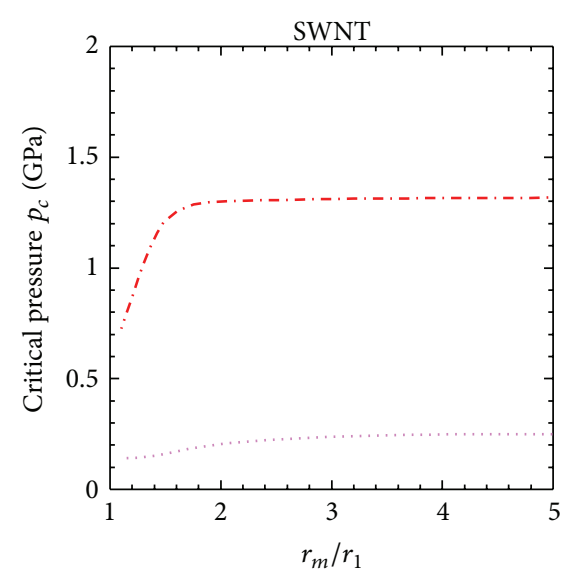

(b)

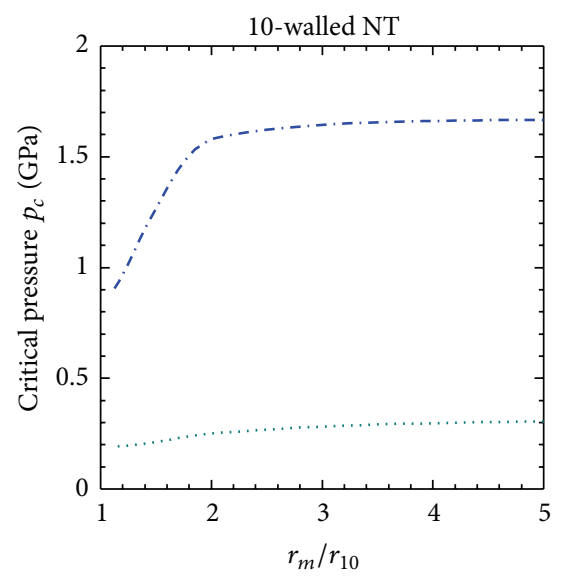

(e)

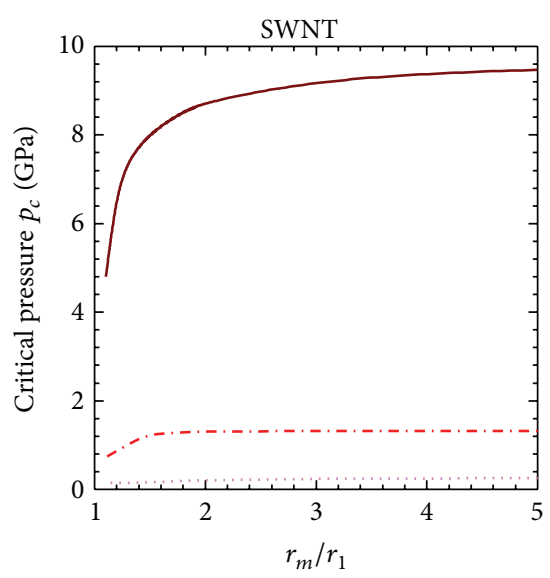

(c)

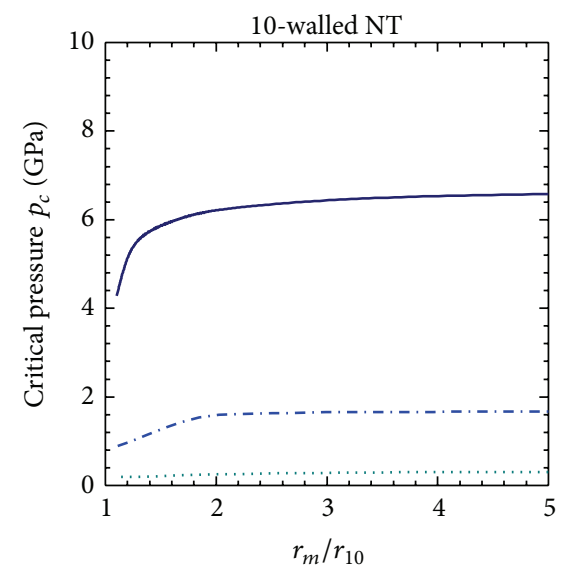

(f)

FiguRE 2: Critical pressure $p_{c}$ for cross-sectional deformation of embedded carbon nanotubes. Monotonic increases with $r_{m} / r_{N}$, the ratio between the radii of the medium $\left(r_{m}\right)$ and the outermost tube $\left(r_{N}\right)$, are observed regardless of the Young's modulus ratio $E_{m} / E$ between the elastic medium $\left(E_{m}\right)$ and the carbon nanotubes $(E): E_{m} / E=10^{-3}$ (dashed curves), $E_{m} / E=10^{-2}$ (dash-dotted curves), and $E_{m} / E=10^{-1}$ (solid curve).

radius $r_{m}$ in units of $r_{N}$. The growth of $p_{c}$ is rapid for $r_{m} / r_{N}<$ 2 , and then, it saturates for larger $r_{m} / r_{N}$. The rapid growth in $p_{c}$ indicates a "hardening effect" caused by the surrounding medium, that is, an enhancement in the radial stiffness of the embedded nanotube by encapsulation. This hardening effect disappears with a further increase in $r_{m} / r_{N}$; the results imply that the surrounding medium with thickness $r_{m}>5 r_{N}$ no longer enhances the hardening effect and thus can be identified as a medium with infinitely large thickness $\left(r_{m} \rightarrow\right.$ $\infty)$, which is the case considered in our earlier work [12].

Figure 3 provides the index of radial deformation modes observed immediately above $p_{c}$. Successive transformation of deformation modes with increasing medium thickness $r_{m}$ was confirmed, as a result of the fact that the energy required to deform the surrounding medium needed to be responsible for determining the stable corrugation pattern of the embedded nanotube. Again, we found that the mode variation disappears for $r_{m} / r_{N}>3$, and a larger value of $n$ arises for a higher modulus ratio $E_{m} / E$. The nonmonotonic variance in the corrugation mode $n$ observed within $1<$ $r_{m} / r_{N}<2$ is what can be clarified for the first time by the present work; it is applicable to the condition $r_{m} \sim r_{N}$, which lay beyond the scope of our earlier work [12].

Figure 4 shows the $p_{c}$ dependence on the modulus ratio $\left(E_{m} / E\right)$, where the radius ratio $\alpha \equiv r_{m} / r_{N}$ is fixed to be $\alpha=$ 1.1 or 5.0. For every choice of $\alpha$ and $N$, the $p_{c}$ curves in this plot obey a power law represented by

$$
p_{c} \propto\left(\frac{E_{m}}{E}\right)^{\beta} \quad \text { with } \beta \simeq \frac{2}{3} \text {. }
$$

We have confirmed that the power-law behaviour holds for any values of $\alpha$ and $N$ within the ranges of $1.1<\alpha<5$ and $1<N<10$, respectively. Recall here that, for $\alpha>5$, $p_{c}$ becomes almost constant even with increasing $\alpha$. These facts lead to the conjecture that the power law described by (38) is universal to all embedded nanotubes, at least under the numerical conditions that we have addressed. The power law of $p_{c}$ represented by (38) is an interesting manifestation of the embedding effect of nanotubes into an elastic medium.

It is worthy to note that the two-third power law of $p_{c}$ has been also proposed for the buckling of macroscopic "tunnels" 


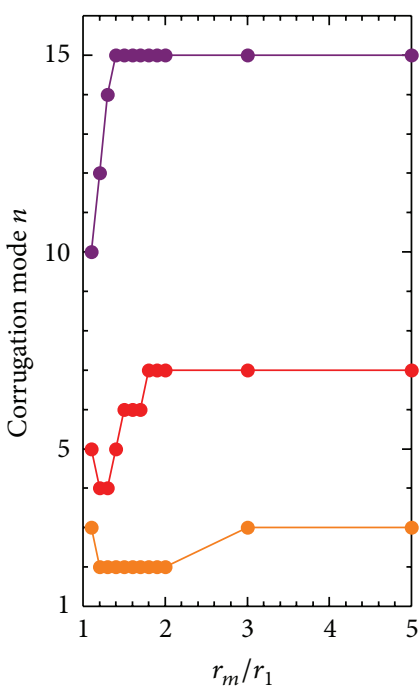

(a) SWNT

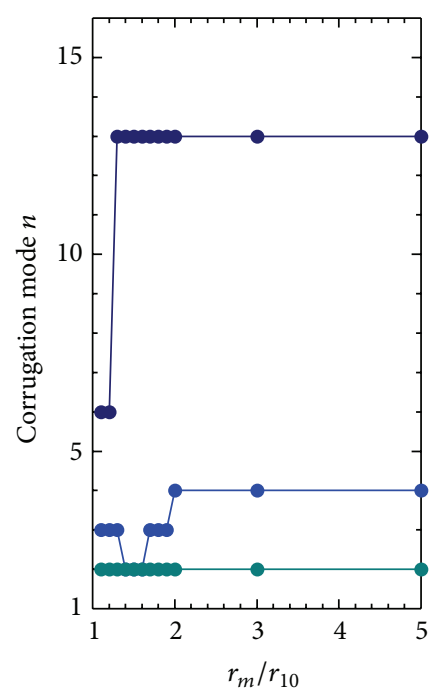

(b) 10-walled NT

FIGURE 3: Mode index $n$ of the radial corrugation observed in the embedded nanotubes. The modulus ratio is set to $E_{m} / E=10^{-3}, 10^{-2}$, and $10^{-1}$ from the bottom to the top.

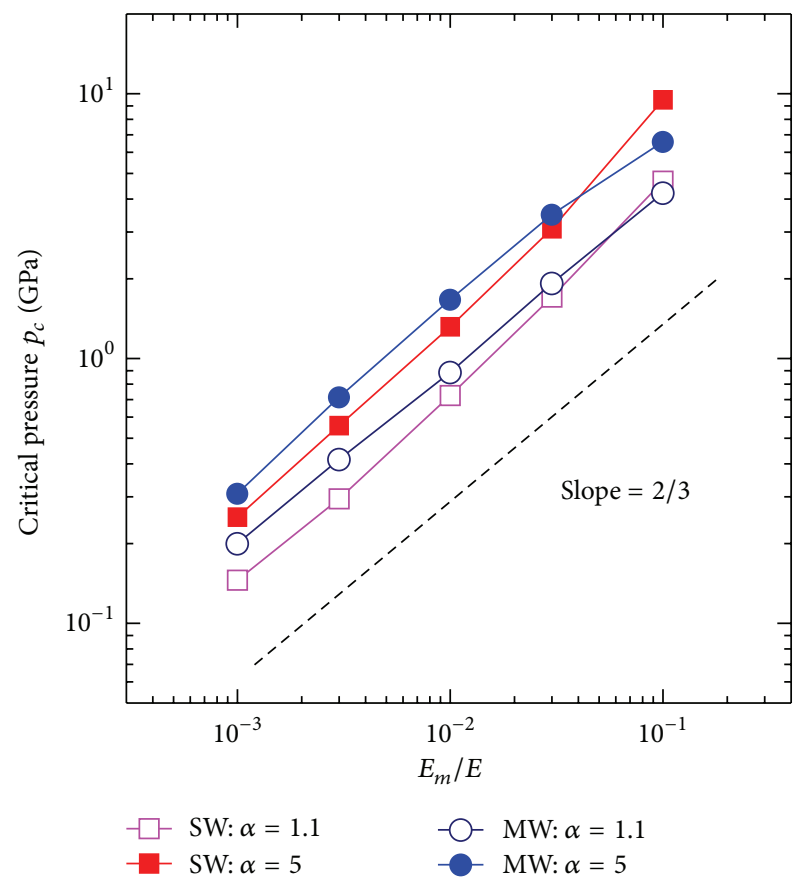

FIGURE 4: Double logarithm plot of the critical pressure $p_{c}$ as a function of the Young's modulus ratio $E_{m} / E$ between the elastic medium $\left(E_{m}\right)$ and the nanotubes $(E)$. The parameter $\alpha \equiv r_{m} / r_{N}$ represents the ratio of the medium radius $r_{m}$ to the outermost tube radius $r_{N}$, where $N=1$ for single-walled nanotubes and $N=10$ for MWNTs. For all the parameter values chosen, the two-third power law of $p_{c}$ given by (38) is observed.

embedded in an infinite soft ground [36]. The radial collapse of tunnel liners, which is one of the major civil engineering disasters, was analysed to reveal a closed form of $p_{c}$; several approximations led to the conclusion of a power-law of $p_{c}$ similar to (38), though an infinitely large medium and only the case of one cylindrical shell (i.e., $N=1$ ) are hypothesized. In this context, our results suggest that the approximation theory proposed for macroscopic tunnels [36] is valid for nanoscopic cylinders, even though they consist of more than one concentric walls.

The previous discussion may pose a further question: does the power law of $p_{c}$ hold true for macroscopic counterparts of "many"-walled nanotubes? In fact, macroscaled pipein-pipe structures (i.e., a pipe inserted inside another pipe) are known to be an efficient design solution for subsea-pipeline developments in deep water $[37,38]$, wherein buckling resistance to large amounts of external hydrostatic pressure is a key structural design requirement. Addressing these problems is expected to shed light on the development of multiplecylindrical structures from multidisciplinary viewpoints and will be considered in our future work.

\section{Conclusion}

We have demonstrated a continuum elastic approach that describes the cross-sectional deformation of carbon nanotubes surrounded by a self-contracting host medium. The approach enables quantitative discussions of the critical pressure for radial corrugation and the stable corrugation mode of the nanotube surrounded by the contracting medium. Numerical calculations based on the established theory revealed the power-law dependence of the critical pressure on the elastic modulus of the medium, which is independent of the medium thickness. Further studies are expected to shed light on other mechanical properties of embedded nanotubes and to suggest applications based on their unique crosssectional deformations. 


\section{Acknowledgments}

This work was supported by JSPS KAKENHI (Grant nos. 24686096, 11J03636, 22760058, and 25390147). One of the authors (H. Shima) appreciates the financial support provided by the Asahi Glass Foundation.

\section{References}

[1] H. Shima and M. Sato, Elastic and Plastic Buckling of Carbon Nanotubes, Pan Stanford Publishing, Singapore, 1st edition, 2013.

[2] H. Shima, "Buckling of carbon nanotubes: a state of the art review," Materials, vol. 5, no. 1, pp. 47-84, 2012.

[3] Z. Osváth, G. Vértesy, L. Tapasztó et al., "Atomically resolved STM images of carbon nanotube defects produced by Ar+ irradiation," Physical Reviev B, vol. 72, no. 4, Article ID 045429, 6 pages, 2005.

[4] A. V. Krasheninnikov and K. Nordlund, "Ion and electron irradiation-induced effects in nanostructured materials," Journal of Applied Physics, vol. 107, no. 7, Article ID 071301, 2010.

[5] P. M. Ajayan, V. Ravikumar, and J. C. Charlier, "Surface reconstructions and dimensional changes in single-walled carbon nanotubes," Physical Review Letters, vol. 81, no. 7, pp. 1437-1440, 1998.

[6] F. Ding, K. Jiao, Y. Lin, and B. I. Yakobson, "How evaporating carbon nanotubes retain their perfection?" Nano Letters, vol. 7, no. 3, pp. 681-684, 2007.

[7] F. Börrnert, S. Gorantla, A. Bachmatiuk et al., "In situ observations of self-repairing single-walled carbon nanotubes," Physical Review B, vol. 81, no. 20, Article ID 201401, 4 pages, 2010.

[8] L. Sun, F. Banhart, A. V. Krasheninnikov, J. A. RodríguezManzo, M. Terrones, and P. M. Ajayan, "Carbon nanotubes as high-pressure cylinders and nanoextruders," Science, vol. 312, no. 5777, pp. 1199-1202, 2006.

[9] Y. Guo and W. Guo, "Reassembly of single-walled carbon nanotubes into hybrid multilayered nanostructures inside nanotube extruders," Physical Review B, vol. 76, no. 4, Article ID 045404, 2007.

[10] Z. Xu, L. Wang, and Q. Zheng, "Enhanced mechanical properties of prestressed multi-walled carbon nanotubes," Small, vol. 4, no. 6, pp. 733-737, 2008.

[11] S. H. Yang, L. L. Feng, and F. Feng, "Torsional behaviour of carbon nanotubes with abnormal interlayer distances," Journal of Physics D, vol. 42, no. 5, Article ID 055414, 5 pages, 2009.

[12] H. Shima, M. Sato, K. Iiboshi, S. Ghosh, and M. Arroyo, "Diverse corrugation pattern in radially shrinking carbon nanotubes," Physical Review B, vol. 82, no. 8, Article ID 085401, 2010.

[13] H. Shima and M. Sato, "Multiple radial corrugations in multiwalled carbon nanotubes under pressure," Nanotechnology, vol. 19, no. 49, Article ID 495705, 2008.

[14] H. Shima and M. Sato, "Pressure-induced structural transitions in multi-walled carbon nanotubes," Physica Status Solidi (A), vol. 206, no. 10, pp. 2228-2233, 2009.

[15] C. Ducati, K. Koziol, S. Friedrichs et al., "Crystallographic order in multi-walled carbon nanotubes synthesized in the presence of nitrogen," Small, vol. 2, no. 6, pp. 774-784, 2006.

[16] X. Huang, W. Liang, and S. Zhang, "Radial corrugations of multi-walled carbon nanotubes driven by inter-wall nonbonding interactions," Nanoscale Research Letters, vol. 6, no. 1, article 53, 6 pages, 2011.
[17] M. Majumder, N. Chopra, R. Andrews, and B. J. Hinds, "Nanoscale hydrodynamics: enhanced flow in carbon nanotubes," Nature, vol. 438, no. 7064, p. 44, 2005.

[18] A. Noy, H. G. Park, F. Fornasiero, J. K. Holt, C. P. Grigoropoulos, and O. Bakajin, "Nanofluidics in carbon nanotubes," Nano Today, vol. 2, no. 6, pp. 22-29, 2007.

[19] M. Whitby and N. Quirke, "Fluid flow in carbon nanotubes and nanopipes," Nature Nanotechnology, vol. 2, no. 2, pp. 87-94, 2007.

[20] E. Frackowiak and F. Béguin, "Electrochemical storage of energy in carbon nanotubes and nanostructured carbons," Carbon, vol. 40, no. 10, pp. 1775-1787, 2002.

[21] C.-K. Yang, J. Zhao, and J. P. Lu, "Magnetism of transitionmetal/carbon-nanotube hybrid structures," Physical Review Letters, vol. 90, no. 25, Article ID 257203, 4 pages, 2003.

[22] Y. Maniwa, K. Matsuda, H. Kyakuno et al., "Water-filled singlewall carbon nanotubes as molecular nanovalves," Nature Materials, vol. 6, no. 2, pp. 135-141, 2007.

[23] H. Shima and H. Yoshioka, "Electronic spectral shift of oxygenfilled $(6,6)$ carbon nanotubes," Chemical Physics Letters, vol. 513, no. 4-6, pp. 224-228, 2011.

[24] S. S. Gupta, F. G. Bosco, and R. C. Batra, "Wall thickness and elastic moduli of single-walled carbon nanotubes from frequencies of axial, torsional and inextensional modes of vibration," Computational Materials Science, vol. 47, no. 4, pp. 1049-1059, 2010.

[25] K. N. Kudin, G. E. Scuseria, and B. I. Yakobson, " $\mathrm{C}_{2}$ F, BN, and C nanoshell elasticity from $a b$ initio computations," Physical Review B, vol. 64, no. 23, Article ID 235406, 10 pages, 2001.

[26] X. Q. He, S. Kitipornchai, and K. M. Liew, "Buckling analysis of multi-walled carbon nanotubes: a continuum model accounting for van der Waals interaction," Journal of the Mechanics and Physics of Solids, vol. 53, no. 2, pp. 303-326, 2005.

[27] W. B. Lu, B. Liu, J. Wu et al., "Continuum modeling of van der Waals interactions between carbon nanotube walls," Applied Physics Letters, vol. 94, no. 10, Article ID 101917, 3 pages, 2009.

[28] H. Shima, S. Ghosh, M. Arroyo, K. Iiboshi, and M. Sato, "Thinshell theory based analysis of radially pressurized multiwall carbon nanotubes," Computational Materials Science, vol. 52, no. 1, pp. 90-94, 2012.

[29] L. A. Girifalco, M. Hodak, and R. S. Lee, "Carbon nanotubes, buckyballs, ropes, and a universal graphitic potential," Physical Review B, vol. 62, no. 19, pp. 13104-13110, 2000.

[30] M. Sammalkorpi, A. Krasheninnikov, A. Kuronen, K. Nordlund, and K. Kaski, "Mechanical properties of carbon nanotubes with vacancies and related defects," Physical Review B, vol. 70, no. 24, Article ID 245416, 8 pages, 2005.

[31] N. M. Pugno, "Young's modulus reduction of defective nanotubes," Applied Physics Letters, vol. 90, no. 4, Article ID 043106, 3 pages, 2007.

[32] A. V. Krasheninnikov and F. Banhart, "Engineering of nanostructured carbon materials with electron or ion beams," Nature Materials, vol. 6, no. 10, pp. 723-733, 2007.

[33] S. Okada, T. Mukawa, R. Kobayashi et al., "Comparison of Young's modulus dependency on beam accelerating voltage between electron-beam- and focused ion-beam-induced chemical vapor deposition pillars," Japanese Journal of Applied Physics Part 1, vol. 45, no. 6 B, pp. 5556-5559, 2006.

[34] A. Champi, A. S. Ferlauto, F. Alvarez, S. R. P. Silva, and F. C. Marques, "On the elastic constants of amorphous carbon nitride," Diamond and Related Materials, vol. 17, no. 11, pp. 18501852, 2008. 
[35] S. R. Timoshenko and J. N. Goodier, Theory of Elasticity, McGraw-Hill, 3rd edition, 1970.

[36] J. G. A. Croll, "Buckling of cylindrical tunnel liners," Journal of Engineering Mechanics, vol. 127, no. 4, pp. 333-341, 2001.

[37] M. Sato and M. H. Patel, "Exact and simplified estimations for elastic buckling pressures of structural pipe-in-pipe cross sections under external hydrostatic pressure," Journal of Marine Science and Technology, vol. 12, no. 4, pp. 251-262, 2007.

[38] M. Sato, M. H. Patel, and F. Trarieux, "Static displacement and elastic buckling characteristics of structural pipe-in-pipe crosssections," Structural Engineering and Mechanics, vol. 30, no. 3, pp. 263-278, 2008. 

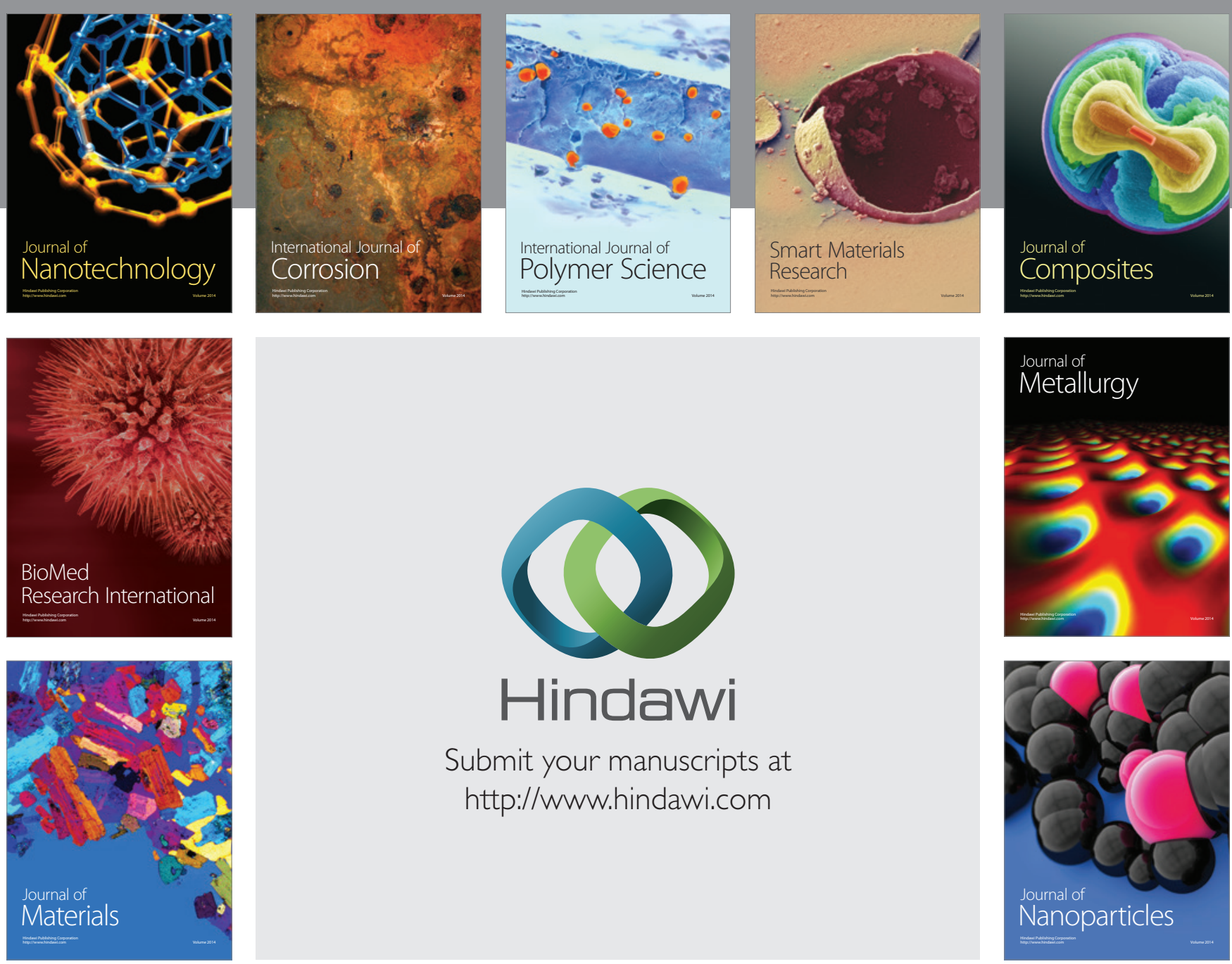

Submit your manuscripts at http://www.hindawi.com
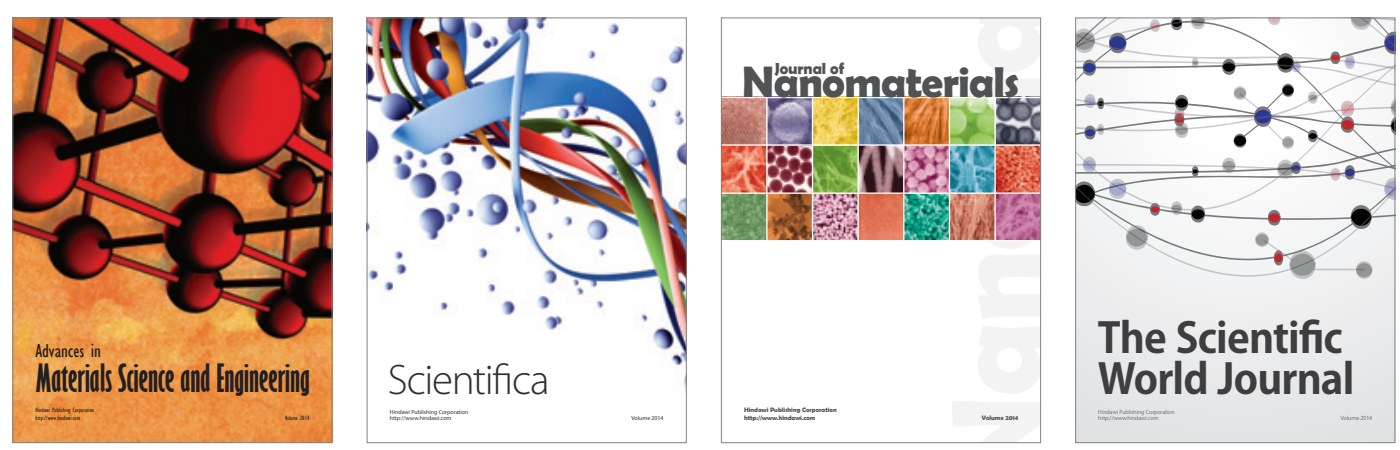

\section{The Scientific World Journal}
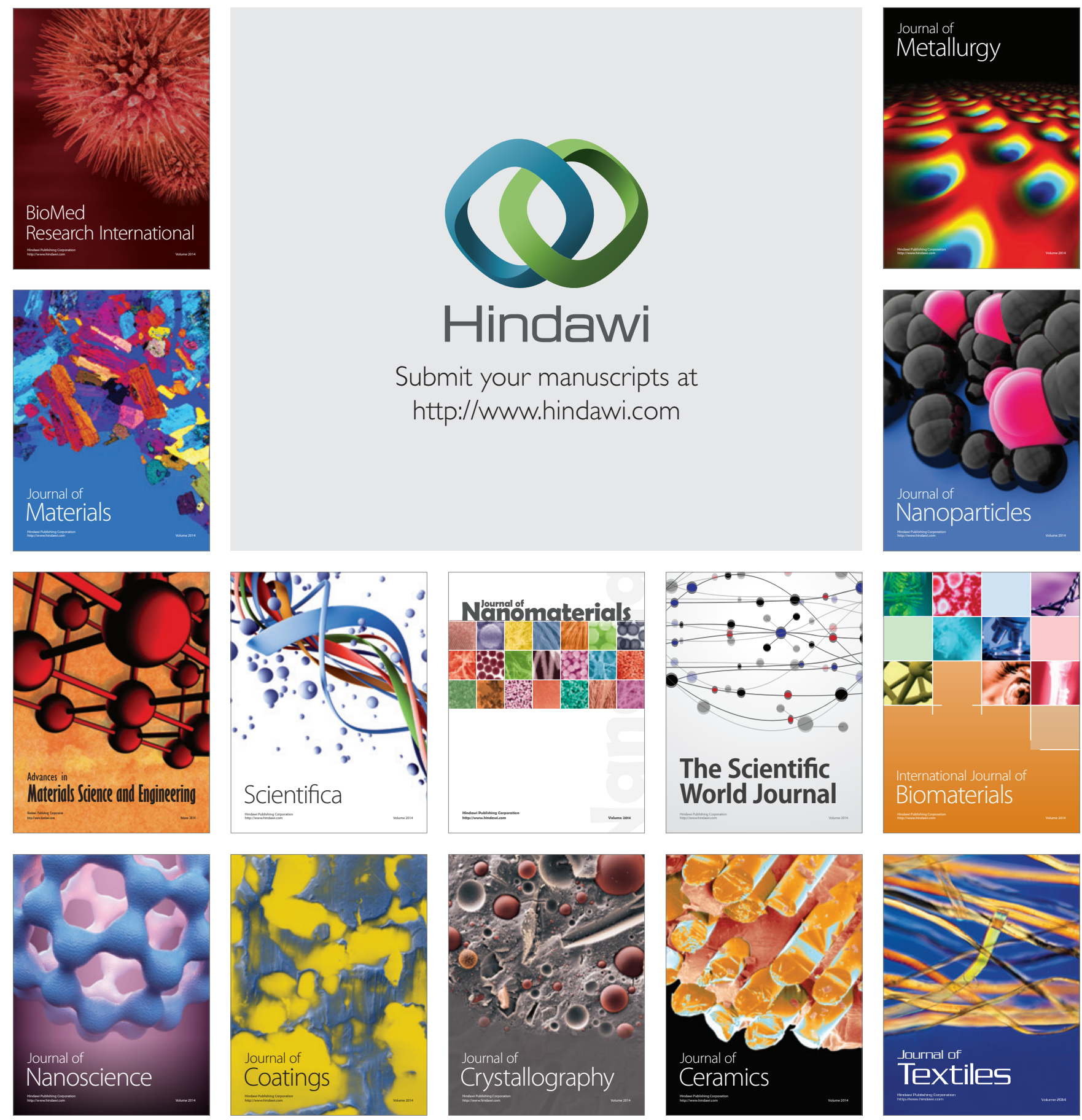